Hydrology and Earth System Sciences, 6(2), 139-151 (2002) C EGS

\title{
Application of a plane-stratified emission model to predict the effects of vegetation in passive microwave radiometry
}

\author{
Khil-ha Lee, R. Chawn Harlow, Eleanor J. Burke and W. James Shuttleworth \\ Department of Hydrology and Water Resources, University of Arizona, Tucson, AZ, USA \\ E-mail of corresponding author: eleanor@hwr.arizona.edu
}

\begin{abstract}
This paper reports the application to vegetation canopies of a coherent model for the propagation of electromagnetic radiation through a stratified medium. The resulting multi-layer vegetation model is plausibly realistic in that it recognises the dielectric permittivity of the vegetation matter, the mixing of the dielectric permittivities for vegetation and air within the canopy and, in simplified terms, the overall vertical distribution of dielectric permittivity and temperature through the canopy. Any sharp changes in the dielectric profile of the canopy resulted in interference effects manifested as oscillations in the microwave brightness temperature as a function of canopy height or look angle. However, when Gaussian broadening of the top and bottom of the canopy (reflecting the natural variability between plants) was included within the model, these oscillations were eliminated. The model parameters required to specify the dielectric profile within the canopy, particularly the parameters that quantify the dielectric mixing between vegetation and air in the canopy, are not usually available in typical field experiments. Thus, the feasibility of specifying these parameters using an advanced single-criterion, multiple-parameter optimisation technique was investigated by automatically minimizing the difference between the modelled and measured brightness temperatures. The results imply that the mixing parameters can be so determined but only if other parameters that specify vegetation dry matter and water content are measured independently. The new model was then applied to investigate the sensitivity of microwave emission to specific vegetation parameters.
\end{abstract}

Keywords: passive microwave, soil moisture, vegetation, SMOS, retrieval

\section{Introduction}

Passive microwave radiometers operating at L-band $(21 \mathrm{~cm}$, $1.4 \mathrm{GHz}$ ) frequencies have promise as tools to measure the moisture of the surface layer of the soil over large areas (Jackson et al., 1999). It is well recognised that the relationship between microwave brightness temperature and soil moisture can be influenced significantly by several factors, including soil properties and - especially significant in the context of the present paper - the nature and form of any overlying vegetation (Wigneron et al., 1998).

A vegetative canopy will scatter and absorb the emission from the soil. It will also contribute its own emission that will be scattered and absorbed by the canopy it passes through. Any downward emission will be reflected by the soil surface and again scattered and absorbed by the canopy. The water held within the canopy is mainly responsible for this scattering, absorption and emission. The two main approaches to account for the effect of vegetation on the microwave emission from the soil are based on solutions of the radiative transfer equation (Kerr and Wigneron, 1994). The simpler, more common approach is valid only at the lower frequencies, where any scattering effects can be assumed to be small. The vegetation is treated as a homogeneous uniform layer and uses two semi-empirical parameters: the optical depth and the single scattering albedo (Ulaby et al., 1986). At L-band, the single-scattering albedo is assumed to be zero (Jackson and Schmugge, 1991) and the optical depth is proportional to the vegetation water content, where the value of the constant of proportionality, the opacity coefficient, is an uncertain function of the canopy type and structure, wavelength, polarisation and, possibly, also vegetation water content (Jackson and Schmugge, 1991; Wigneron et al., 1996, 2000; Le Vine and Karam, 1996). An alternative approach is to create a complex model that 
includes a detailed description of all of the components of the canopy (Wigneron et al., 1993; Burke et al., 1999), where all the required input parameters are measured properties of the canopy. However, this model requires a very detailed description of the vegetative canopy. This paper discusses an alternative approach - the development of a realistic model of intermediate complexity that captures the more important features of a vegetative canopy, but requires fewer input parameters. This type of model might prove to be highly appropriate for in-field validation studies and for evaluating the reliability of satellite retrieval algorithms that necessarily make simplifying assumptions concerning the vegetation, e.g., ESA's Soil Moisture and Ocean Salinity (SMOS) upcoming L-band mission (Wigneron et al., 2000).

The model evaluated in this paper is an extension of the Wilheit (1978) model for the coherent propagation of electromagnetic radiation through a stratified medium; it is applied frequently to model microwave emission from soils (Burke et al., 1997, 1998). However, in this paper, it is used to describe the combined emission from both the soil and vegetation. This application is highly suitable for use in coupled land surface and microwave emission models such as that used by Burke et al. (1997, 1998). In fact, in this example, the Common Land Model (CLM, 2000) land surface scheme is used to provide soil profile information required by the Wilheit (1978) model.

Unfortunately, in no field experiments to date have all the vegetation parameters required as input by the extended Wilheit (1978) model been measured. However, multiparameter optimisation techniques have been shown (Bastidas et al., 1999; Burke et al., 2002; Sen et al., 2001) to be very powerful tools in the estimation of model parameters. This paper discusses the potential of using multiparameter, single-criterion techniques to evaluate the values of the parameters required by the extended Wilheit (1978) model.A companion paper (Lee et al., 2002) uses this extended Wilheit (1978) model to provide a realistic description of the vegetation canopy when creating synthetic microwave brightness temperatures for the SMOS mission. It then examines the ability of the proposed SMOS retrieval to obtain the soil water content, vegetation water content and effective temperature from these data.

\section{Modelling approach}

Two types of model are used to predict microwave emission from soils: coherent (Wilheit, 1978; Njoku and Kong, 1977; England, 1976) and non-coherent (Burke et al., 1979; England, 1975). Both Schmugge and Choudhury (1981) and Ulaby et al. (1986) compared coherent and non-coherent emission models and their results are summarised below. Non-coherent models estimate emissivity using the dielectric contrast at the air/soil interface and are accurate only when the (variable) sampling depth within the soil is well known. In coherent models, the value of the emissivity at the surface is coupled to the dielectric properties below the surface; hence, they provide better estimates of the emissivity when the soil water content profile is non-uniform. One disadvantage of coherent models is that interference effects can occur. However, these have been witnessed only rarely in nature (Schmugge et al., 1998). The Wilheit (1978) model was selected for this study because of its accuracy in nonuniform conditions and it was configured to minimise the impact of interference. Because it does not include scattering effects, it can be used only in conditions where scattering is negligible, i.e. at L-band.

The Wilheit (1978) model assumes that the medium under consideration consists of a layered dielectric of semi-infinite extent, that the boundaries between the layers are planar and parallel and that each layer is homogenous. To calculate the instantaneous microwave emission, it is also assumed that each layer is in thermal equilibrium, so that each layer of dielectric emits the same amount of energy as it absorbs. The microwave brightness temperature, $T_{B}$, can then be expressed as:

$$
T_{B}=\sum_{i=1}^{N} f_{i} T_{i}
$$

where $T_{i}$ is the temperature of the $i^{\text {th }}$ layer, $f_{i}$ is the fraction of energy absorbed from an incident microwave by the $i^{t h}$ layer (a function of the dielectric constant of the soil), and $N$ is the number of layers in the semi-infinite medium.

The dielectric and temperature profiles of the soil, required as input by the Wilheit (1978) model, were predicted using results from the Common Land Model (CLM, 2000) land surface scheme. The CLM includes the best elements of some well-tested physical parameterisations and numerical schemes (e.g., LSM, Bonan, 1996; BATS, Dickinson et al., 1986, and IAP, Dai and Zeng, 1997). It requires a set of parameters that specify physical constants and aspects of the soil and vegetation and, given meteorological forcing variables, it then provides prognostic and diagnostic landsurface state variables and surface energy and water fluxes as output. CLM describes the diffusion of soil heat and liquid water through several (typically ten) layers of soil using the finite difference form of the thermal diffusion equation and Richard's equation, respectively. CLM uses the fraction of sand and clay to specify the properties of the soil. If vegetation is present, it is represented as though it is at a single level, at the height $(d+z 0)$, where $d$ is the zero plane displacement and $z 0$ is the aerodynamic roughness of the 
canopy. Turbulent transport between this level and a specified reference height above the canopy is described using the Monin-Obukhov similarity theory, with allowance made for the effect of atmospheric stability. The whole canopy surface resistance is parameterised following the model introduced by Jarvis (1976).

Following Burke et al. (1997) and Camillo et al. (1986), the soil component of the microwave emission model was run with 200 layers at the depths indicated: $0-1 \mathrm{~cm}, 10$ layers at $1 \mathrm{~mm} ; 1-3 \mathrm{~cm}, 10$ layers at $2 \mathrm{~mm} ; 3--6 \mathrm{~cm}, 10$ layers at $3 \mathrm{~mm} ; 6-10 \mathrm{~cm}, 10$ layers at $4 \mathrm{~mm} ; 1-20 \mathrm{~cm}, 20$ layers at $5 \mathrm{~mm}$; and $20-160 \mathrm{~cm}, 140$ layers at $10 \mathrm{~mm}$. The soil temperature and soil water content output by the CLM for the ten layers simulated in this study were linearly interpolated to the 200 soil layers required by the microwave emission model.

The Wilheit (1978) model calculates the depth below which the emission is negligible and neglects all layers below this depth. Because this depth is a function of soil type and soil water content profile, it may well change every time step. The Dobson et al. (1985) model is used to predict the profile of soil dielectric from knowledge of the profile of soil water content and soil particle size distribution. This is a simple non-linear dielectric-mixing model that takes into account the proportions of free water (dielectric $\sim 80$ ), bound water (dielectric $\sim 4$ ), soil solids (dielectric $\sim 4$ ) and air (dielectric $\sim$ ) within the soil medium. The soil particle size distribution determines the relative proportions of free and bound water.

The extended Wilheit (1978) model also requires the dielectric and temperature profile within the vegetation. Limited information is available in the literature about the dielectric permittivity of either the vegetative matter (ElRayes and Ulaby, 1987; Ulaby and Jedlicka, 1984; Chuah et al., 1997; Colpitts and Coleman, 1997; Franchois et al., 1998; Ulaby et al., 1986) or the canopy itself (Ulaby et al., 1986; Ulaby and Jedlicka, 1984; Brunfeldt and Ulaby, 1984; Schmugge and Jackson, 1992). To model the dielectric properties of a vegetative canopy, two separate mixing effects must be taken into account. The first mixing is that between the constituents of the vegetation to get the dielectric of the vegetative matter itself. The approach to modelling the dielectric of the vegetative matter taken in this paper is analogous to a linear version of the Dobson et al. (1985) mixing model for soils:

$$
\varepsilon_{v}=\varepsilon_{d r y} V_{d r y}+\varepsilon_{f w} V_{f w}+\varepsilon_{b w} V_{b w}
$$

where $\varepsilon_{v}$ is the dielectric permittivity for leaf material as a whole, $\varepsilon_{d r y}, \varepsilon_{f w}$, and $\varepsilon_{b w}$ are the dielectric permittivities, and $V_{d r y}, V_{f w}$, and $V_{b w}$ are the volume fractions of dry matter, free water and bounded water, respectively. It is assumed that $\varepsilon_{d r y}, \varepsilon_{f w}$, and $\varepsilon_{b w}$ are independent of the vegetation water content. The dielectric permittivity of the mixed vegetation matter and air making up a vegetation canopy $\left(\varepsilon_{\text {can }}\right)$ is given by:

$$
\varepsilon_{\text {can }}^{\alpha}=\varepsilon_{v}^{\alpha} V_{v}+\varepsilon_{\text {air }}^{\alpha}\left(1-V_{v}\right)
$$

where $\varepsilon_{a i r}$ is the dielectric permittivity of air; $V_{v}$ is the fractional volume of vegetation elements per unit volume canopy; and $\alpha$ is a so-called "shape factor". (In the case of the Dobson et al., 1985 mixing model for soils, $\alpha=0.65$.) Schmugge and Jackson (1992) suggested that the refractive model $(\alpha=0.5)$ provides a better representation of the dielectric properties of the canopy than a linear model $(\alpha=1)$. However, the dielectric permittivity of a canopy is very similar to air and very much lower than that of soil, and there is little additional evidence to indicate the value of $\alpha$ for such a sparse medium. One of the purposes of this present study was to investigate the feasibility of deriving values of this parameter from field data.

In initial studies, the profile of dielectric within the canopy was assumed to be uniform. However, despite the dielectric of the canopy being close to air, this resulted in interference effects. Interference occurs when discrete changes in dielectric permittivity are assumed at two or more surfaces, e.g. at the top or bottom of the canopy as well as at the soil surface. In this case, a portion of the microwaves incident on each surface will be reflected and add constructively or destructively. Consequently, the modelled radiometric temperature changes greatly, depending on the thickness of the canopy, the wavelength of the radiation and the angle of emission. This phenomenon has been observed only as a wetting front descends within the soil (Schmugge et al., 1998) and is unlikely to be observed in vegetation because there is never a discrete and uniform change at the top or bottom of the vegetative canopy. The natural variability between the individual plants that make up the canopy results in a more gradual change. The effect of this natural variability was modelled using Gaussian broadening at the top and bottom of the canopy. The Gaussian broadening was introduced so that the total amount of dielectric within the canopy remained constant but it was redistributed over a slightly greater height. The Appendix describes in more detail how the vertical profiles of canopy dielectric permittivity are specified in the model. Figure 1 shows an example of the interference effects which occur in the modelled microwave brightness temperature as a function of look angle when assuming a discrete change at the canopy top $(0.9 \mathrm{~m})$ and bottom $(0.09 \mathrm{~m})$ rather than a more realistic canopy with Gaussian broadening of both the top and bottom 


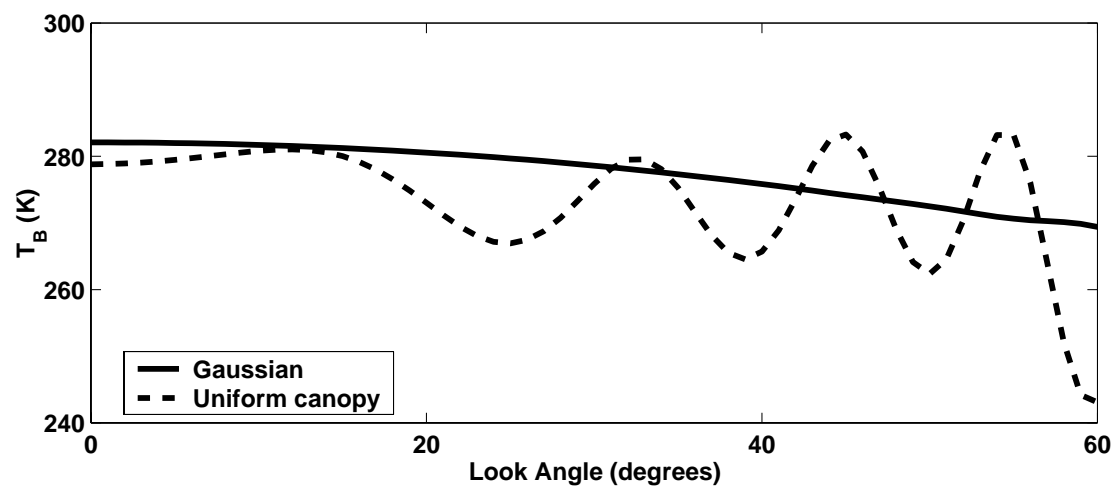

Fig. 1. The modelled brightness temperature as a function of observation angle calculated at 11:30 a.m. on DOY 208 using the DRYDOWN2 data set with and without Gaussian smoothing of the top and bottom of the canopy.

(with a standard deviation of $0.09 \mathrm{~m}$ in each case). In this example, the canopy was assumed to consist of 100 layers, each $1 \mathrm{~cm}$ thick. This amount of detail is required to smooth the interfaces at the top and bottom of the canopy sufficiently. For the range of look angles shown, it is evident that Gaussian broadening eliminates the effect of withincanopy internal reflections and interference. It should be noted that, even with gradual changes in dielectric permittivity, significant reflections may still occur at very shallow angles. However, for the observation angles normally used by field and satellite systems, this reflection phenomenon and its associated interference are effectively suppressed.

The temperature of the canopy is a prognostic variable in CLM. In the present study, the temperature profile of the canopy used within the microwave emission model is estimated very simply. For canopy above $\left(d+z_{0}\right)$ - where $d$ is the zero plane displacement and $z_{0}$ is the aerodynamic roughness of the canopy - it is estimated by linear interpolation between the air temperature at the reference height and the simulated canopy temperature. For canopy below $\left(d+z_{0}\right)$, it is estimated by linear interpolation between the simulated canopy temperature and the simulated soilsurface temperature below. It is also assumed that the description of aerodynamic transfer assumed in CLM is insensitive to the detailed shape of the canopy dielectric profile specified in the microwave emission model.

\section{Optimisation for evaluating model}

\section{parameters}

Bastidas et al. (1999) and Sen et al. (2001) provided a detailed discussion of the usefulness of multi-criteria optimisation methods for the evaluation and improvement of land-surface schemes. For example, they showed that the use of calibration procedures may lead to reductions of 20 to $30 \%$ in the root mean square error (RMSE) between model and measurements when compared to the traditional method of using lookup tables for model parameter estimation. In addition, they gained valuable insight into acceptable ranges of model parameters, the quality of the model physics and the complexity of the model required to describe a particular scenario. The present study uses a single-criterion optimisation technique to minimize the difference between time series of measured and modelled microwave brightness temperatures and explores the feasibility of estimating the values of the vegetation parameters in the extended Wilheit (1978) microwave emission model.

In general, a numerical model might have $n$ parameters (in this case, the parameters describing the dielectric profile of the vegetation) to be calibrated using $m$ observations (in this case, the time series of microwave brightness temperatures). The distance between the $m$ model-simulated responses and the $m$ observations is defined by an objective function $(O)$ such as the root mean square error between the modelled responses and observations (RMSE). The goal of a model calibration is then to find the preferred value for the $n$ parameters within the feasible set of parameters that minimise $O$. The Shuffled Complex Evolution algorithm (SCE-UA, Duan et al., 1993, 1994) is a single-criterion, multi-parameter optimisation technique developed at the University of Arizona. It is a general-purpose global optimisation method designed to handle many of the response surface problems encountered in the calibration of nonlinear simulation models. It randomly samples the feasible parameter space to select a population of points. The population is then partitioned into several "complexes", each of which evolves independently in a manner based on the downhill simplex algorithm (Nelder and Mead, 1965). 
The population is periodically "shuffled" and new complexes formed so that the information gained by previous complexes is shared. As the search progresses, the entire population tends to converge towards the neighbourhood of the global optimum value for the objective function. These steps are repeated until prescribed termination rules are satisfied (Duan et al., 1993, 1994).

The SCE-UA has been used successfully to calibrate, against field observations, the values of the parameters in a hydrological model (Duan et al., 1993, 1994). In addition, Burke et al. (2002) used it to retrieve near surface soil moisture and vegetation water content for several patches of different land-cover types within one pixel using a set of brightness temperatures measured simultaneously at a wide range of look angles. The new application discussed here
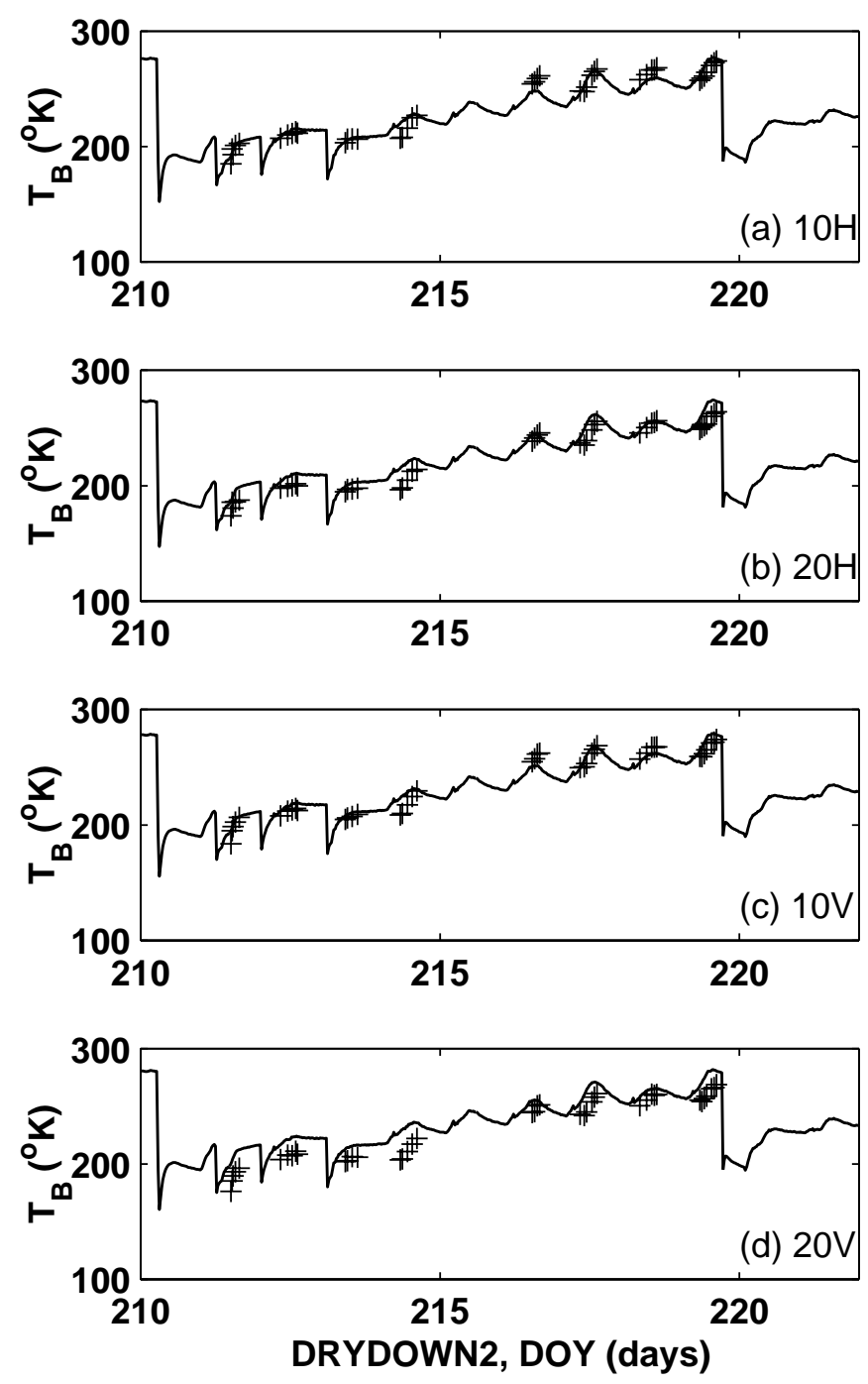

explores the feasibility of estimating parameters within a microwave emission model.

\section{Field data}

The experimental data used in this paper were collected over a soybean canopy monitored during a field experiment carried out in 1985 at the USDA, ARS Beltsville Agricultural Research Center, Maryland, USA. ( Burke et al., 1998).

The site consisted of a $10 \times 10 \mathrm{~m}$ controlled plot containing loamy sand soil. Half of the plot was left bare and half was covered with soybeans. Different periods of drying were monitored during the soybean growth, with the bare soil monitored at the same time. A dual-polarised L-band (21-
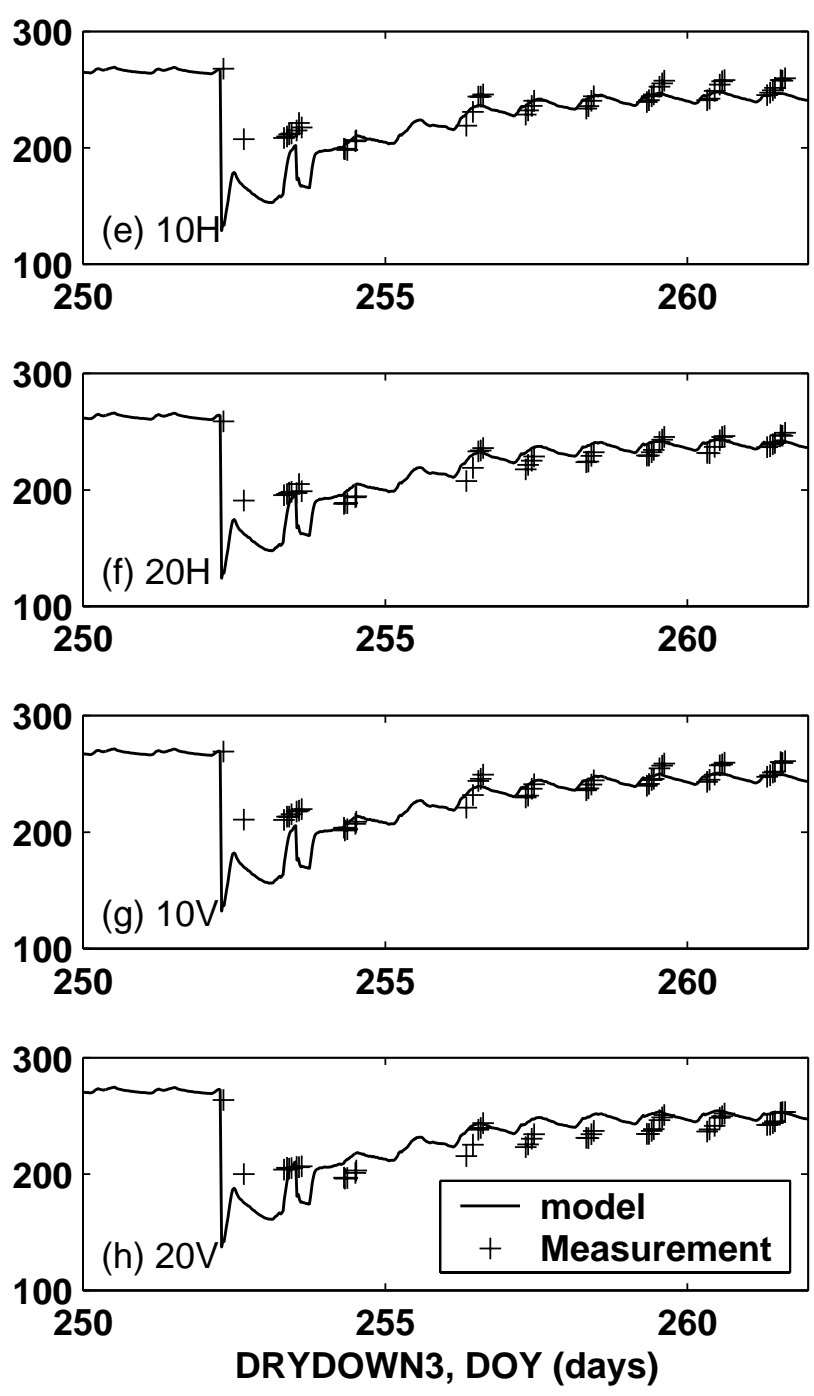

Fig. 2. Time series of measured and modelled microwave brightness temperatures for a bare soil surface during DRYDOWN2 and DRYDOWN3. 10 and 20 denote $10^{\circ}$ and $20^{\circ}$ look angles, and $H$ and $V$ denote horizontal and vertical polarisation, respectively. 
cm wavelength, 1.4 GHZ frequency) passive microwave radiometer measured the horizontally and vertically polarised microwave brightness temperatures at view angles of $10^{\circ}$ and $20^{\circ}$. In addition to the radiometric measurement, soil and limited vegetation characteristics (including singlepoint volumetric soil-water content, soil temperature, the hydraulic properties of the soil, plant height, and the wet and dry matter weight of the vegetation) were measured. The data from the Beltsville site used in this study fall into two distinct periods that are hereafter referred to as DRYDOWN2, between Day of Year (DOY) 210 and 222, and DRYDOWN3, between DOY 252 and 262.

\section{Model results}

\section{SOIL MICROWAVE EMISSION}

One of the assumptions in using SCE-UA to optimise the vegetation parameters in the extended Wilheit (1978) model for soil and vegetation, is that there is a high level of agreement between the modelled and measured microwave brightness temperatures at the soil surface. CLM was used to simulate surface energy and water fluxes and the related changes in soil temperature and soil moisture for a bare soil and, hence, calculate the dielectric permittivity profile of the soil for input into the bare soil Wilheit (1978) model. Figure 2 shows the microwave brightness temperature predicted using the time series of profile soil water content and soil temperature predicted by CLM for DRYDOWN2 and DRYDOWN3 for a bare soil. There are some discrepancies on the days immediately after irrigation; however, as the soil dries out further, there is excellent agreement between the modelled and measured brightness temperatures.

The ability of CLM to describe relevant changes in nearsurface soil temperature and soil moisture under a vegetation canopy was also evaluated. Figure 3 shows the soil temperature at $1-\mathrm{cm}$ depth and the average soil moisture over the depth ranges $0-2 \mathrm{~cm}$ and $0-5 \mathrm{~cm}$ as modelled by CLM during DRYDOWN2 and DRYDOWN3 using the soil parameters measured at the site and vegetation parameters for IGBP vegetation cover class 12 ["cropland"]. The equivalent measured values are also shown in this figure when available. The RMSE for DRYDOWN2 is $2.1{ }^{\circ} \mathrm{C}$ for temperature at $1 \mathrm{~cm}$ depth, $3.1 \%$ for $0-2 \mathrm{~cm}$ average soil
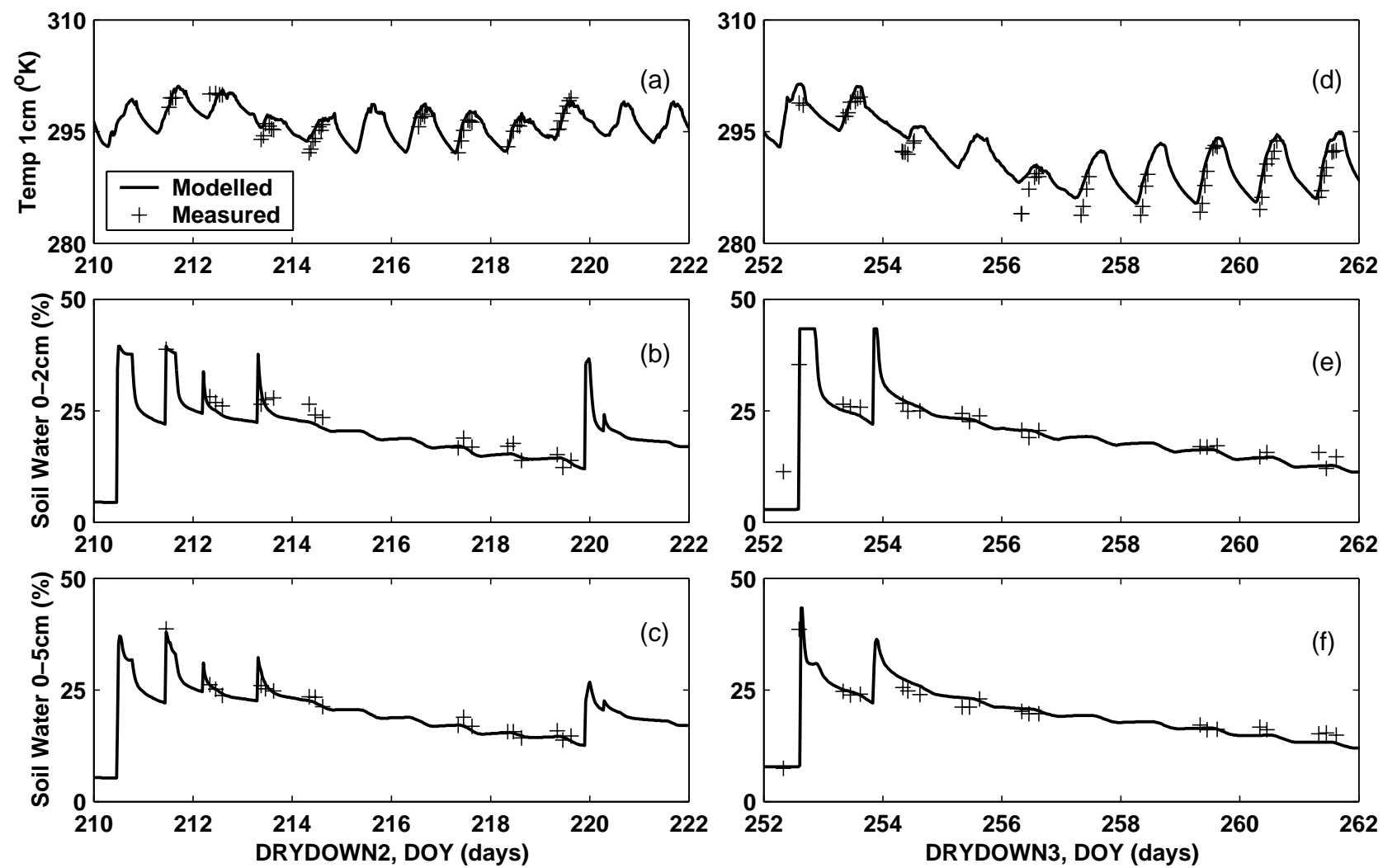

Fig. 3. CLM-calculated and observed values of soil temperature at $1 \mathrm{~cm}$ depth (a and d); average soil moisture over 0-2 $\mathrm{cm}$ depth ( $b$ and $e$ ); and 0-5 cm depth (c and f) for DRYDOWN2 (a, b, and $c$ ) and DRYDOWN3 (d, e, and f). 
moisture, and $2.1 \%$ for $0-5 \mathrm{~cm}$ average soil moisture. The RMSE for DRYDOWN3 is $2.6^{\circ} \mathrm{C}$ for temperature at $1 \mathrm{~cm}$ depth, $2 \%$ for $0-2 \mathrm{~cm}$ average soil moisture, and $1.9 \%$ for $0-5 \mathrm{~cm}$ average soil moisture. The time series of both the modelled temperature and soil moisture show good agreement with the measurements.

Given that CLM can model, accurately, the near surface soil water content and the near surface temperature under a vegetation canopy, and that the Wilheit (1978) model for bare soil predicts, accurately, the microwave brightness temperature for a bare soil, it can be assumed that the extended Wilheit (1978) model for soil and vegetation will predict, accurately, the microwave emission at the soil surface.

\section{EXTENDED WILHEIT (1978) MODEL FOR SOIL AND VEGETATION}

The extended Wilheit (1978) model for soil and vegetation was run within the SCE algorithm and the RMSE between the modelled and measured microwave brightness temperature minimised by optimising the parameters that describe the dielectric profile of the canopy. These parameters are salinity ( $S$ in ppt); canopy height $(t$ in m); height of bottom of canopy ( $b$ in $\mathrm{m})$; standard deviation of top height $\left(\sigma_{t}\right.$ in $\left.\mathrm{m}\right)$; standard deviation of bottom height $\left(\sigma_{b}\right.$ in $\left.\mathrm{m}\right)$; fresh weight of the canopy $\left(V_{w}\right.$ in $\left.\mathrm{kg} \mathrm{m}^{-2}\right)$; volume fraction of free water $\left(V_{f w}\right.$ in $\left.\mathrm{m}^{3} \mathrm{~m}^{-3}\right)$; volume fraction of dry matter $\left(V_{d r y}\right.$ in $\left.\mathrm{m}^{3} \mathrm{~m}^{-3}\right)$ and dielectric mixing coefficients for vertical and horizontal polarisation $\left(\alpha_{V}\right.$ and $\left.\alpha_{H}\right)$. Optimum values were found for the DRYDOWN2 and DRYDOWN3 periods separately by minimising the RMSE between the observed and modelled microwave brightness temperature at L-band and both $\left(10^{\circ}\right.$ and $\left.20^{\circ}\right)$ observation angles. It should be noted that, when operating in a complex parameter space, multi-parameter optimisation algorithms (including SCE) discover "local" minima of the objective function as well as the required "global" minimum. Therefore, the SCEUA algorithm was randomly initiated and run 20 times for each example creating 20 different parameter sets. The parameter set that provides the minimum RMSE was then selected to be the "preferred parameter set". Figure 4 shows
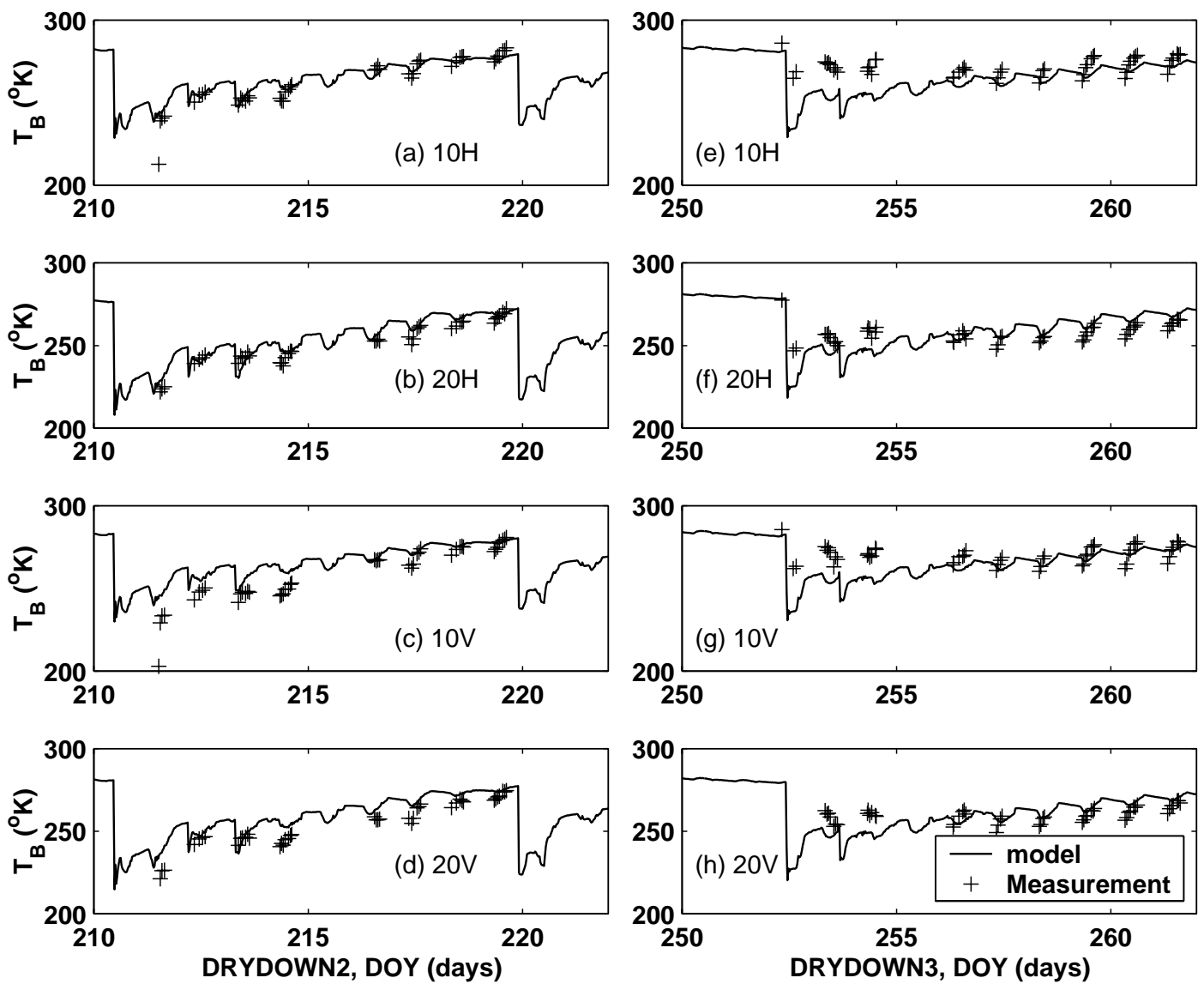

Fig. 4. Time series of measured and modelled microwave brightness temperatures in the presence of vegetation during DRYDOWN2 and DRYDOWN3. 10 and 20 denote $10^{\circ}$ and $20^{\circ}$ look angles, and $H$ and $V$ denote horizontal and vertical polarisation, respectively. 
an example of the excellent agreement between model and measured time series of microwave brightness temperatures.

Unfortunately, for this example, there is insufficient information in the available measured microwave brightness temperatures to use single-criterion multi-parameter optimisation techniques to determine the parameters that control the shape of the canopy (height of bottom $(b)$ and the standard deviation of the Gaussian broadening of top $\left(\sigma_{t}\right)$ and bottom $\left.\left(\sigma_{b}\right)\right)$. This may be partly because the observations were made at two angles fairly close to the zenith. However, it also implies that, at least for look angles near the nadir, the microwave brightness temperature predicted by this model is insensitive to canopy structure. (Note: from the standpoint of retrieving estimates of soil moisture and vegetation optical depth from remotely sensed observations, it is helpful that the canopy structure is modelled to have only a limited influence on microwave surface brightness temperature because precise knowledge of canopy structure will usually not be available for use in the retrieval process.)

In the next example, the values of these three parameters, $b, \sigma_{b}$, and $\sigma_{t}$, were arbitrarily set to be $15 \%$ of the height of the canopy, this being a plausible value that avoids the model-generated interference effects at observation angles of $10^{\circ}$ and $20^{\circ}$, and the simulations repeated. Despite the lack of sensitivity of the model to these canopy shape parameters (and hence the high likelihood of SCE-UA generating any value of these parameters within the allowed range), it was reassuring to see that the optimised values of the other parameters in this new simulation were very similar to those found previously. However, there was also interaction between some pairs of vegetation-related parameters that have opposing influence on the overall microwave emission by a vegetation canopy. This made it difficult to obtain independent estimates of these parameters merely from a time series of observations of microwave brightness temperature at just two look angles. Fortunately, some of the relevant parameters were measured during the field experiment, specifically: the fresh weight of the vegetation (3.42 and $4.41 \mathrm{~kg} \mathrm{~m}^{-2}$ during DRYDOWN2 and DRYDOWN3, respectively) and the crop height (0.89 and $1.15 \mathrm{~m}$ during DRYDOWN2 and DRYDOWN3, respectively). The volume fraction of dry matter $\left(V_{d r y}\right)$ was calculated $(0.38$ and 0.5 during DRYDOWN2 and DRYDOWN3 respectively) from the mass fraction of dry matter by assuming a dry matter density of $0.3 \mathrm{~g} \mathrm{~cm}^{-3}$ (Ulaby et al., 1986). The parameters remaining to be determined by multi-parameter optimisation are the volume fraction of free water $\left(V_{f w}\right)$, salinity of the water in the vegetation $(S)$, and the mixing coefficient $(\alpha)$ defined in Eqn. (3). Since $\alpha$ may well be a function of polarisation, different values were assumed for the vertically and horizontally polarised brightness temperature data $\left(\alpha_{H}\right.$ and $\left.\alpha_{V}\right)$.

Table 1 lists the optimised values of vegetation-related parameters for the soybean canopy at the Beltsville site during DRYDOWN2 and DRYDOWN3. The values shown

Table 1. Parameters used in the coupled model and their values for the Beltsville site as given by multi-parameter optimisation (unless pre-set to fixed values prior to optimisation).

\begin{tabular}{|c|c|c|c|c|c|c|c|c|c|c|}
\hline \multirow{2}{*}{$\begin{array}{l}\text { Parameter } \\
\text { Fractional free water } \\
\text { volume in vegetation } \\
\left(\mathrm{m}^{3} \mathrm{~m}^{-3}\right)\end{array}$} & \multirow{2}{*}{$\begin{array}{l}\text { Symbol } \\
\text { name }\end{array}$} & \multirow{2}{*}{$\begin{array}{l}\begin{array}{l}\text { Range of } \\
\text { optimisation }\end{array} \\
0-1 \\
\begin{array}{l}\text { accurately } \\
\text { optimised) }\end{array}\end{array}$} & \multicolumn{2}{|c|}{$\begin{array}{l}\text { Values during } \\
\text { DRYDOWN2 }\end{array}$} & \multicolumn{2}{|c|}{$\begin{array}{l}\text { Values during } \\
\text { DRYDOWN3 }\end{array}$} & \multicolumn{2}{|c|}{$\begin{array}{l}\text { Values during } \\
\text { DRYDOWN2 }\end{array}$} & \multicolumn{2}{|c|}{$\begin{array}{l}\text { Values during } \\
\text { DRYDOWN3 }\end{array}$} \\
\hline & & & 0.041 & 0.040 & 0.048 & 0.047 & $\begin{array}{l}0.5 \\
\text { (fixed) }\end{array}$ & $\begin{array}{l}0.5 \\
\text { (fixed) }\end{array}$ & $\begin{array}{l}0.5 \\
\text { (fixed) }\end{array}$ & $\begin{array}{l}0.5 \\
\text { (fixed) }\end{array}$ \\
\hline $\begin{array}{l}\text { Salinity of water in } \\
\text { vegetation } \\
\text { (ppt) }\end{array}$ & S & $\begin{array}{l}0-15 \\
\text { (when } \\
\text { optimised) }\end{array}$ & $\begin{array}{l}0 \\
\text { (fixed) }\end{array}$ & 0.00 & $\begin{array}{l}0 \\
\text { (fixed) }\end{array}$ & 0.00 & $\begin{array}{l}0 \\
\text { (fixed) }\end{array}$ & 0.94 & $\begin{array}{l}0 \\
\text { (fixed) }\end{array}$ & 0.73 \\
\hline $\begin{array}{l}\text { Mixing parameter for } \\
\text { L-band with } \\
\text { horizontal polarisation } \\
\text { (dimensionless) }\end{array}$ & $\alpha_{L}$ & $0-3$ & 1.98 & 1.98 & 1.94 & 1.93 & 1.24 & 1.12 & 1.17 & 1.09 \\
\hline $\begin{array}{l}\text { Mixing parameter for } \\
\text { L-band with } \\
\text { vertical polarisation } \\
\text { dimensionless }\end{array}$ & $\alpha_{H}$ & $0-3$ & 2.07 & 2.07 & 2.19 & 2.18 & 1.29 & 1.16 & 1.31 & 1.22 \\
\hline
\end{tabular}



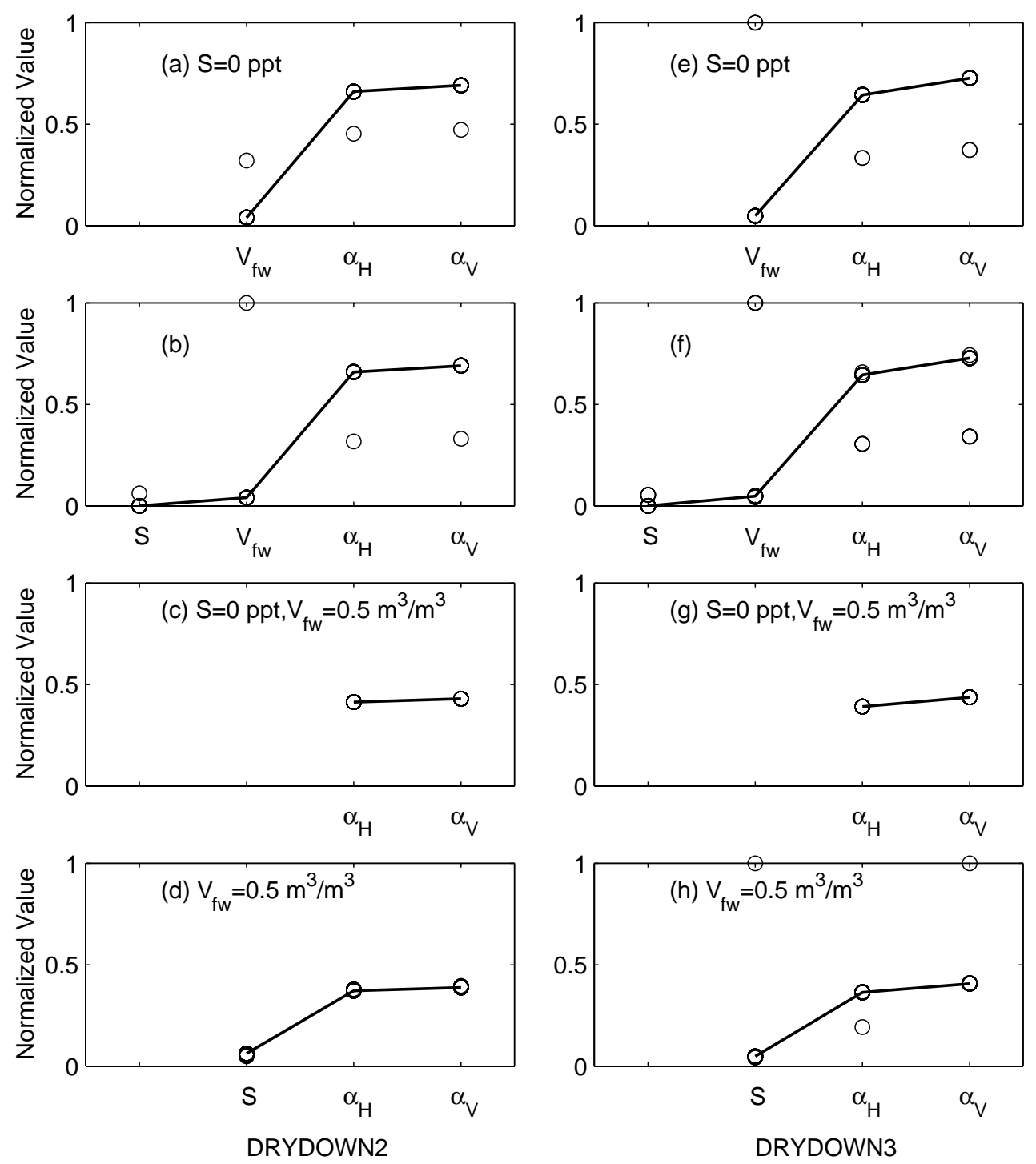

Fig. 5. Estimated parameter values for the 20 replicate optimisations. In each case, parameter values are normalised by the predefined range of each parameter over which optimisation was made, and the set of parameters corresponding to the lowest error in modelled microwave brightness temperature are linked by a line. Results are shown for $\mathrm{S}$ set to 0 ppt ( $a$ and $e$ ); all parameters optimised ( $b$ and f); $\mathrm{S}$ set to 0 ppt and $\mathrm{V}_{\mathrm{fw}}$ set $0.5 \mathrm{~m}^{3} \mathrm{~m}^{-3}$ (c and $\mathrm{g}$ ); and $\mathrm{V}_{\mathrm{fw}}$ set $0.5 \mathrm{~m}^{3} \mathrm{~m}^{-3}$ (d and $h$ ), for DRYDOWN2 (a-d) and DRYDOWN3 $(e-h)$.

are the sets of parameters that produce the minimum RMSE in brightness temperatures from 20 randomly initiated replications: this is assumed to be the global minimum parameter set. Also shown is the predefined allowed range of each parameter. Eight optimisation runs were made. In the first four, the fractional free water volume, $V_{f w}$, was optimised along with other parameters. Because this resulted in optimised values for $V_{f w}$ that were unexpectedly low when compared to the typically cited value, the value of $V_{f w}$ in the second four optimisations was (arbitrarily) set to the more typical value of 0.5 . In each group of four optimisation runs, two optimisations were made for DRYDOWN2 and two for DRYDOWN3, with the value of salinity optimised in one and set to 0 parts per thousant (ppt) in the other. Figure 5 demonstrates the potential range of these optimised parameters by showing the optimised parameter values normalised by the predefined allowed range of each optimised parameter for the 20-member sample of local minima identified by the SCE algorithm during the optimisation runs. In each figure, the normalised values for the parameter set with the smallest objective function (assumed to be the global minimum) are linked. In general, SCE-UA repeatedly sampled a single parameter set which corresponded to a well-defined global minimum value for 
the RMSE. In all cases, the parameter set with minimum RMSE fell within the predefined range of selection. However, in a few cases (Figs. 5b, e, f, h), the multiparameter optimisation process became trapped in subsidiary minimum and defined parameter sets with an RMSE significantly greater than that of the global minimum and with one or more parameters at the limit of the allowed range.

Some features of the optimisation results are obvious and consistent. All of the optimisation runs in which the salinity, $S$, of the water in the vegetation was optimised gave values for this parameter that were very close to $0 \mathrm{ppt}$. The four runs in which the fractional fresh water volume, $V_{f w}$, was not prescribed resulted in similar, low, optimised values $(\sim 0.04-0.05)$ for this parameter, and the values of $\alpha_{H}$ and $\alpha_{V}$ simultaneously estimated by multi-parameter optimisation were markedly different and larger than the corresponding values found when $V_{f w}$ was set to (the more plausible) value of 0.5 during optimisation. It is clear that there is a strong correlation between the value of $V_{f w}$ and the apparent strength of in-canopy dielectric mixing as expressed in the optimised values of the $\alpha$ parameters. This point is discussed further in the context of the singleparameter sensitivity described in the next section. Notwithstanding the interdependence of parameters just mentioned, for a given value of $V_{f w}$, whether optimised or prescribed, it is reassuring that the preferred values of $\alpha_{H}$ and $\alpha_{\mathrm{v}}$ are fairly similar in DRYDOWN2 and DRYDOWN3. The values of $\alpha_{H}$ and $\alpha_{V}$ are slightly different, with the value for vertical polarisation higher in all cases, suggesting some differences between the effect of the canopy at horizontal and vertical polarisation. It should be noted that the values of $\alpha$ retrieved by the optimisation procedure are higher than the values found by Schmugge and Jackson (1992). However, little other information is available on the realistic range of $\alpha$. One possible error in the determination of $\alpha$ could be that some of the other parameters were set to slightly wrong values and the optimisation procedure adjusted itself accordingly.

\section{SENSITIVITY TO INDIVIDUAL MODEL PARAMETERS}

It is of interest to investigate the sensitivity of microwave brightness temperature to the assigned values of parameters in the extended Wilheit (1978) model. To illustrate this, Fig. 6 shows how the calculated brightness temperature viewed at an angle of $20^{\circ}$ at 11:30 p.m. on DOY 224 varies for a range of parameter values around the optimised values for
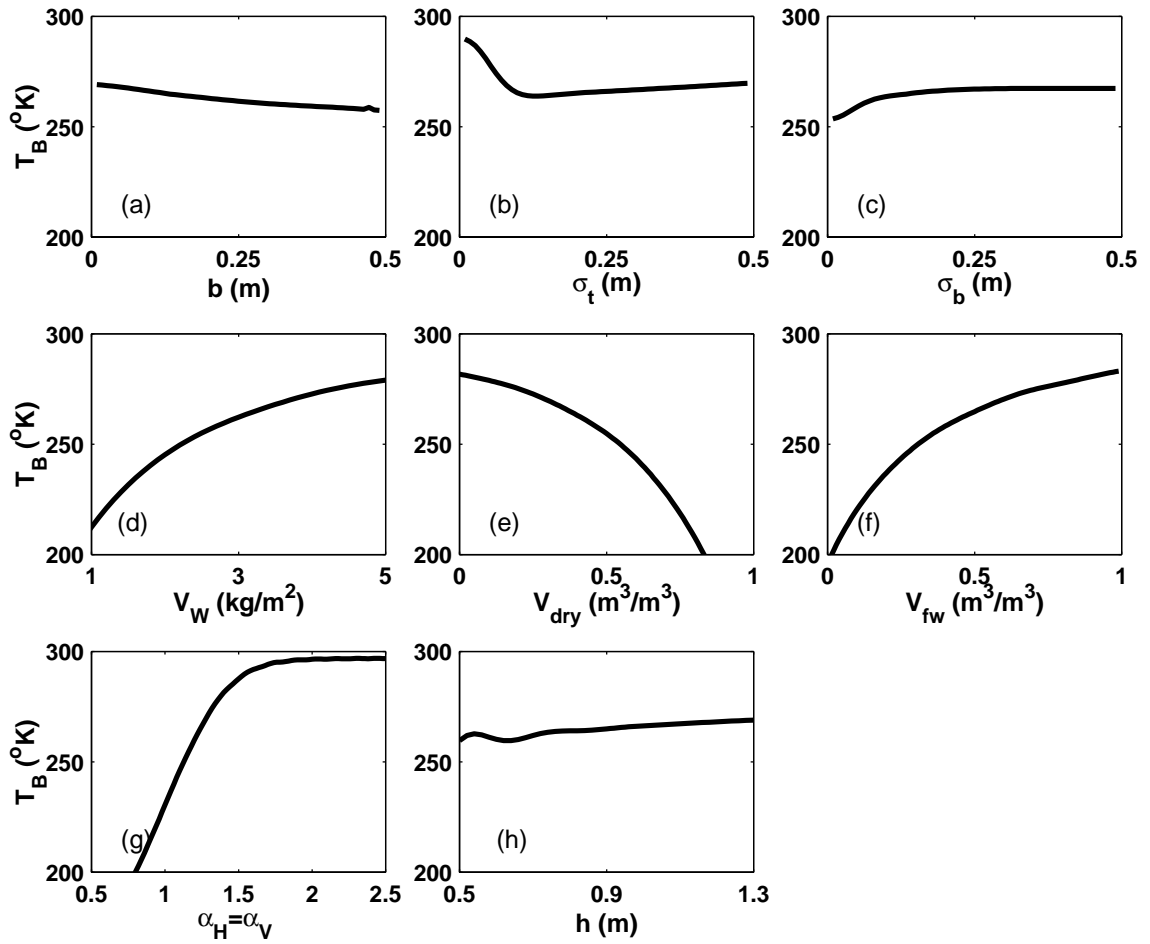

Fig. 6. Modelled microwave brightness temperature for a range of values of individual parameters around their optimised value (parameter specified in the $x$-axis), with the salinity of in-canopy water set to zero. The calculations are made at 11:30 p.m. on DOY 224. 
L-band microwave radiation. The calculations are made using the optimised values for DRYDOWN2, with the fractional free water volume and salinity of the vegetation fixed at 0.5 and $0 \mathrm{ppt}$, respectively (Fig. $5 \mathrm{c}$ ). The sensitivity to three groups of model parameters, those controlling the shape of the canopy, the composition of the vegetation, and the extent of in-canopy dielectric mixing, are shown in Fig. 6 for dry soil conditions.

Figures $6 \mathrm{a}-\mathrm{c}$ demonstrate the expected result that calculated microwave emission is only weakly related to the value of the model parameters that control the shape of the canopy over a broad range of values. However, at very low values of $\sigma_{t}$ and $\sigma_{b}$, there is evidence of higher sensitivity. This is because, when the transition in dielectric permittivity at the top/bottom of the canopy becomes very sharp, some modelled within-canopy internal reflection (and constructive/destructive interference) occurs even for a view angle of $20^{\circ}$. Figures $6 \mathrm{~d}-\mathrm{f}$ show, as expected, that the fresh weight of the canopy $\left(V_{w}\right)$ can have a significant impact on modelled microwave brightness temperatures. The extent of in-canopy dielectric mixing, as represented by the value of $\alpha$, directly influences the overall value of the canopy dielectric permittivity. Consequently, the sensitivity to the value of a shown in Fig. $5 \mathrm{~g}$ is to be anticipated.
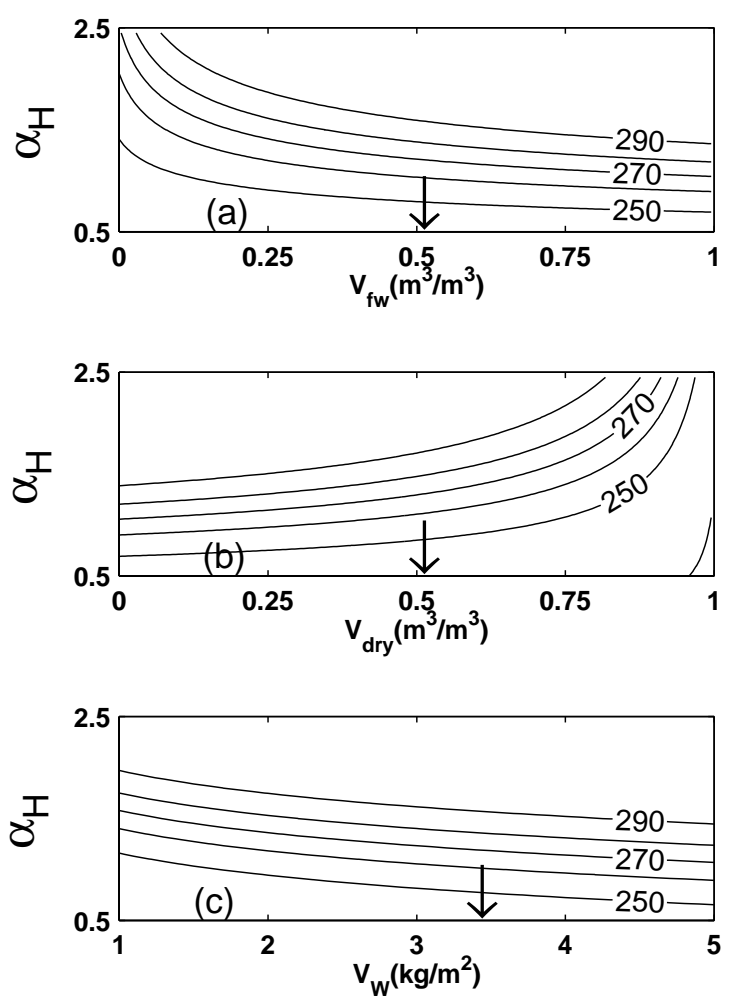

Fig. 7. Contours of the horizontally polarised modelled microwave brightness temperature at nadir at 11:30 p.m. on DOY 224 using the optimised parameter given in column 8 of Table 1. Calculations are made for combinations of $\alpha_{\mathrm{H}}$ and $\mathrm{V}_{\mathrm{fw}}(a), \alpha_{\mathrm{H}}$ and $\mathrm{V}_{\mathrm{dry}}(b)$, and $\alpha_{\mathrm{H}}$ and $\mathrm{V}_{\mathrm{w}}(c)$.
Because non-linear dielectric mixing (i.e. the values of $\alpha$ ) and vegetation composition parameters (especially $V_{w}$ and $\left.V_{f w}\right)$ together determine the overall canopy dielectric permittivity, it will always be difficult and perhaps impossible to determine their values in the model by multiparameter optimisation methods from a time series of the observed microwave brightness temperature. Figure 7 illustrates this point by showing contours of the modelled microwave brightness temperature, $T_{B}$, at a look angle of $20^{\circ}$ at the Beltsville site calculated at 11:30 p.m. on DOY 224 using the optimised parameter given in column 8 of Table 1, but for a range of values of $\alpha$ and a range of values of $V_{f w}, V_{d r y}$, or $V_{w}$.

In the context of field studies of the microwave emission of vegetation in general and in future field validation studies associated with the SMOS and Advanced Microwave Scaning Radiometer (AMSR) missions in particular, it is strongly recommended that these key vegetation composition parameters be measured directly. Doing so will allow an opportunity to investigate the significance of in-canopy dielectric mixing for the vegetation under study. This, in turn, will allow more detailed investigation of the relationship between the optical depth at microwave frequencies of sampled canopies and their vegetation water content, the latter being a potentially more valuable geophysical variable.

\section{Discussion and conclusions}

This paper describes the application of the Wilheit (1978) model for the coherent propagation of electromagnetic radiation through a stratified medium to predict the effect of a vegetation canopy on the microwave emission from the soil surface. The model produced a realistic simulation of the microwave emission, provided there was a gradual change in the dielectric at the top and bottom of the canopy as would be associated with the natural variability between plants.

The Common Land Model was used to predict the profiles of soil temperature and water content and vegetation temperature for use within the extended Wilheit (1978) model. These predictions compared favourably with available measurements. However, not all of the relevant vegetation parameters required by the extended Wilheit (1978) model were measured. Therefore, an evaluation of the ability of automatic calibration procedures to predict the required vegetation parameters was performed. Unfortunately, there was not sufficient information in the available range of microwave brightness temperatures to predict all of the required vegetation parameters using multiparameter optimisation methods; this was either because the 
brightness temperature was insensitive to a particular parameter or because there were interactions between parameters such that a high value of one combined with a low value of another produced the same answer as a low value of the former combined with a high value of the latter. However, when any available measurements were included to limit the number of parameters to be determined, and the canopy shape parameters (parameters to which the modelled microwave brightness temperatures are insensitive) set to plausible values, multi-parameter optimisation techniques provided estimates of the remaining parameters-those which would be difficult to measure in any future field experiment. This is particularly relevant for the mixing coefficient $(\alpha)$ between vegetation matter and air used to calculate the dielectric of the vegetation canopy.

Sensitivity studies with the extended Wilheit (1978) model showed that the calculated microwave emission is insensitive to the values of parameters that specify the shape of the canopy, unless these imply a canopy with very rapid changes of dielectric permittivity at the canopy boundaries. Microwave emission is sensitive to parameters that specify the amount of free water in the vegetation and the extent of dielectric mixing in the canopy, which act together to change the whole-canopy dielectric permittivity.

On the basis of the above results, it is recommended that, future field activity for validating the SMOS mission, care be taken to make independent measurements of vegetation composition parameters in addition to measurements of microwave brightness and forcing variables. The extended Wilheit (1978) model, whose development and application is the main subject of this paper, could then be used to investigate in-canopy dielectric mixing effects within canopies and, in this way, the relationship between microwave brightness temperature and vegetation water content could be understood better.

\section{Acknowledgements}

Primary support for Khil-ha Lee and Dr. Eleanor Burke while preparing this paper was provided from NOAA project NA96GP0412. Additional support for Dr. James Shuttleworth was provided from NASA project NAG81531. The editorial assistance provided by Corrie Thies is appreciated.

\section{References}

Bastidas, L.A., Gupta, H.V., Sorooshian, S., Shuttleworth, W.J. and Yang, Z.L.,1999. Sensitivity analysis of a land surface scheme using multicriteria methods. J. Geophys. Res.-Atmos., 104, 19481-19490.
Bonan, G.B., 1996. A land surface model [LSM version 1.0] for ecological, hydrological, and atmospheric studies: technical description and user's guide. NCAR Technical Note, NCAR/ TN-417+STR, Boulder, CO.

Brunfeldt, D.R. and Ulaby, F.T., 1984. Measured microwave emission and scattering in vegetation canopies. IEEE Trans. Geosci. Remote Sens., 22, 520-524.

Burke, E.J., Gurney, R.J., Simmonds, L.P. and O’Neill, P.E., 1998. Using a modeling approach to predict soil hydraulic properties from passive microwave measurements. IEEE Trans. Geosci. Remote Sens., 36, 454-462.

Burke, E.J., Bastidas, L.A. and Shuttleworth, W.J., Multi-patch retrieval for the SMOS mission. IEEE Trans Geosci. Remote Sens. In press.

Burke, E.J., Gurney, R.J., Simmonds, L P. and Jackson, T.J., 1997. Calibrating a soil water and energy budget model with remotely sensed data to obtain quantitative information about the soil. Water Resour. Res., 33, 1689-1697.

Burke, E.J., Wigneron, J.-P. and Gurney, R.J., 1999. The comparison of two models that determine the effects of a vegetation canopy on passive microwave emission. Hydrol. Earth Syst. Sci., 3, 439-444.

Burke, W.J., Schmugge, T.J. and Paris, J.F., 1979. Comparison of 2.8 and $21 \mathrm{~cm}$ microwave radiometer observations over soils with emission model calculations. J. Geophys. Res., 84, 287294.

Camillo, P.J., O’Neill, P.E. and Gurney, R.J., 1986. Estimating soil hydraulic parameters using passive microwave data. IEEE Trans. Geosci. Remote Sens., 24, 930-936.

Chuah, H.T., Kam, S.W. and Chye, Y.H., 1997. Microwave dielectric properties of rubber and oil palm leaf samples: measurement and modelling. Int. J. Remote Sens., 18, 26232639.

Colpitts, B.G. and Coleman, W.K., 1997. Complex permittivity of the potato leaf during imposed drought stress. IEEE Trans. Geosci. Remote Sens., 35, 1059-1064.

Common Land Model, 2000. http://clm.gsfc.nasa.gov.

Dai, Y.J. and Zeng, Q.C., 1997. A land surface model [IAP94] for climate studies, I: formulation and validation in off-line experiments. Adv. Atmos.Sci., 14, 433-460.

Dickinson, R.E., Henderson-Sellers, A., Kennedy, P.J. and Wilson, M.F., 1986. Biosphere-Atmosphere Transfer Scheme [BATS] for the NCAR Community Climate Model. Technical Note, NCAR/ TN-275+STR, National Center for Atmospheric Research, Boulder, CO.

Dobson, M.C., Ulaby, F.T., Hallikainen, M.T. and El-Rayes, M.A., 1985. Microwave behavior of wet soil, 2: dielectic mixing models. IEEE Trans. Geosci. Remote Sens., 23, 35-46.

Duan, Q.Y., Gupta, V.K. and Sorooshian, S., 1993. Shuffled Complex Evolution approach for effective and efficient global minimization. J. Optimiz. Theor. Appl., 76, 501-521.

Duan, Q.Y., Sorooshian, S. and Gupta, V.K., 1994. Optimal use of the SCE-UA global optimization method for calibrating watershed models. J. Hydrol., 158, 265-284.

El-Rayes, M.A. and Ulaby, F.T., 1987. Microwave dielectric spectrum of vegetation, 1: experimental observations. IEEE Trans. Geosci. Remote Sens., 25, 541-549.

England, A.W., 1975. Thermal microwave emission from a scattering layer. J.Geophys. Res., 32, 4484-4496.

England, A.W., 1976. Relative influence upon microwave emissivity of fine-scale stratigraphy. Pure Appl.Geophys., 114, 287-299.

Franchois, A., Pineiro, Y. and Lang, R.H., 1998. Microwave permittivity measurements of two conifers. IEEE Trans. Geosci..Remote Sens., 36, 1384-1395. 
Jackson, T.J. and Schmugge, T.J., 1991. Vegetation effects on the microwave emission of soils. Remote Sens. Environ., 36, 203212.

Jackson, T.J., Le Vine, D.M., Hsu, A.Y., Oldak, A., Starks, P.J., Swift, C.T., Isham, J.D. and Haken, M., 1999. Soil moisture mapping at regional scales using microwave radiometry: the Southern Great Plains hydrology experiment. IEEE Trans. Geosci. Remote Sens., 37, 2136-2151.

Jarvis, P.G., 1976. Interpretation of variations in leaf water potential and stomatal conductance found in canopies in field. Phil. Trans. Roy. Soc., London, Series B, 273, 593-601.

Kerr, Y.H. and Wigneron, J.P., 1994. Vegetation models and observations: a review. In: ESA/NASA International Workshop on Passive Microwave Remote Sensing Research Related to Land-Atmosphere Interaction, B.J. Choudhury, Y.H. Kerr, E.G. Njoku and P. Pampaloni (Eds.), 317-344,

Le Vine, D.M. and Karam, M.A., 1996. Dependence of attenuation in a vegetation canopy on frequency and plant water content. IEEE Trans. Geosci. Remote Sens., 34, 1090-1096.

Lee, K.H., Burke, E.J., Shuttleworth, W.J. and Harlow, R.C., 2002. Influence of vegetation on SMOS mission retrievals. Hydrol. Earth System Sci., 6, 15-28.

Nelder, J.A. and Mead, R.A., 1965. A simplex method for function minimization. Comput. J., 7, 308-313.

Njoku, E.G. and Kong, J.A., 1977. Theory for passive microwave sensing of near surface soil moisture. J. Geophys. Res., 82, 3108-3118.

Schmugge, T.J. and Choudhury, B.J., 1981. A comparison of radiative transfer models for predicting the microwave emission from soils. Radio Sci., 16, 927-938.

Schmugge, T.J. and Jackson, T.J., 1992. A dielectric model of the vegetation effects on the microwave emission from soils. IEEE Trans. Geosci. Remote Sens., 30, 757-760.

Schmugge, T.J., Jackson, T.J., O’Neill, P.E. and Parlange, M.B., 1998. Observations of coherent emissions from soils. Radio Sci., 33, 267-272.

Sen, O.L., Bastidas, L.A., Shuttleworth, W.J., Yang, Z.L., Gupta, H.V. and Sorooshian, S., 2001. Impact of field-calibrated vegetation parameters on GCM climate simulations. Quart. J. Roy. Meteorol. Soc., 127, 1199-1223.

Ulaby, F.T. and Jedlicka, R.P., 1984. Microwave dielectricproperties of plant materials. IEEE Trans. Geosci. Remote Sens., 22, 406-415.

Ulaby, F.T., Moore, R.K. and Fung, A.K., 1986. Microwave Remote Sensing: Active and Passive, Volume 3. Artech House, Inc., 610 Washington St., Dedham, MA.

Wigneron, J.-P., Calvet, J.-C., Kerr, Y.H., Chanzy, A.C. and Lopes, A., 1993. Microwave emission of vegetation, sensitivity to leaf characteristics. IEEE Trans.Geosci.Remote Sens., 31, 716-726.

Wigneron, J.-P., Calvet, J.-C. and Kerr, Y.H., 1996. Monitoring water interception by crop fields from passive microwave observations. Agr. Forest Meteorol. 80, 177-194.

Wigneron, J.-P., Schmugge, T.J., Chanzy, A.C., Calvet, J.-C. and Kerr, Y.H., 1998. Use of passive microwave remote sensing to monitor soil moisture. Agronomy, 18, 27-43.
Wigneron, J.-P., Waldteufel, P., Chanzy, A.C. and Calvet, J.-C., 2000. Two-dimensional microwave interferometer retrieval capabilities over land surfaces. Remote Sens. Environ., 73, 270 282.

Wilheit, T.T., 1978. Radiative transfer in a plane stratified dielectric. IEEE Trans. Geosci. Electron., 16, 138-143.

\section{APPENDIX}

\section{SPECIFICATION OF SMOOTHED DIELECTRIC PROFILES}

The better to represent natural variability in the height of the top and bottom of vegetation canopies, a smooth transition is introduced using Gaussian smoothing. If $t$ and $b$ are the height of top and bottom of the canopy, respectively, and the natural variability in $t$ and $b$ are $\sigma_{\mathrm{t}}$ and $\sigma_{\mathrm{b}}$, respectively, then the normalised through-canopy profile of dielectric permittivity used in the microwave emission model, $\varepsilon_{c}(z)$, is calculated from the function:

$$
\varepsilon_{z}(z)=\varepsilon_{\text {canopy }} \cdot \frac{W_{b}(z) \cdot W_{t}(z)}{\int_{0}^{\infty}\left[W_{b}(z) \cdot W_{t}(z)\right] d z}
$$

where $\varepsilon_{\text {canopy }}$ is the dielectric permittivity of all the leaf material present in the canopy, and the weighting functions, $W_{b}(z)$ and $W_{t}(z)$, are zero for $z<0$ but, for values of $z \geq 0$, are given by:

$$
W_{b}(z)=\frac{\int_{0}^{z} \exp \left(-\left(z^{\prime}-b\right)^{2} / 2 \sigma_{b}^{2}\right) d z^{\prime}}{\int_{0}^{\infty} \exp \left(-\left(z^{\prime}-b\right)^{2} / 2 \sigma_{b}^{2}\right) d z^{\prime}}
$$

and

$$
W_{b}(z)=1-\frac{\int_{0}^{z} \exp \left(-\left(z^{\prime}-t\right)^{2} / 2 \sigma_{t}^{2}\right) d z^{\prime}}{\int_{0}^{\infty} \exp \left(-\left(z^{\prime}-t\right)^{2} / 2 \sigma_{t}^{2}\right) d z^{\prime}}
$$


